\title{
Fact or Fiction: Maximal Image Quality with Minimal Dwell Time
}

\author{
Patrick Trampert $^{1,2}$, Tim Dahmen ${ }^{1}$ and Philipp Slusallek ${ }^{1,2}$ \\ 1. German Research Center for Artificial Intelligence GmbH (DFKI), Saarbrücken, Germany. \\ 2. Saarland University, Saarbrücken, Germany
}

Image quality in scanning electron microscopy is highly dependent on the maximal feasible acquisition time for a dataset. In particular, three-dimensional or large field-of-view imaging suffer from high shot noise, which results in low signal-to-noise ratios and excessive acquisition times. We compared three different strategies to reduce the average dwell time per pixel. We fixed the available acquisition time per frame to make the strategies comparable in terms of maximal possible image quality. The evaluated methods were (1) raster scanning with a reduced dwell time per pixel followed by a state-of-the-art Denoising algorithm, (2) raster scanning with a decreased resolution in conjunction with a state-of-theart Super Resolution (SR) algorithm, and (3) a sparse scanning approach where a fixed percentage of pixels is visited by the beam in combination with state-of-the-art inpainting algorithms. Additionally, increased beam currents were considered for each strategy.

Reducing the dwell time per pixel leads to a decreased number of electrons and, hence, to more shot noise. Acquisitions with this strategy must be denoised to enhance image quality. We applied GeOmetric Analysis operator Learning (GOAL) [1] to the acquired images of strategy one. GOAL uses prior knowledge in the form of a maximally sparse representation learned over an appropriate training set, called dictionary, to solve a convex optimization problem applying a conjugate gradient method. Rather than reducing dwell times, decreasing the number of pixels by increasing the pixel size, as applied in strategy two, saves acquisition time. As this leads to a discretization with bigger step size, image resolution is lost. SR algorithms can compensate for this and increase the resolution to the original size. The applied SR method is based on bilateral Total Variation priors and uses a hierarchical Bayesian framework to uniquely approximate the high resolution image [2]. The third suggested approach reduces the number of pixels by scanning less pixels. Pseudo-random sampling is applied to acquire a small subset of all pixels. Inpainting algorithms are then used to reconstruct the dense data. Besides interpolation, we evaluated algorithms inspired by compressed sensing, like Beta Process Factor Analysis (BPFA) [3] and GOAL, as well as Exemplar-Based Inpainting (EBI) [4]. Orthogonally to these strategies, we investigated the effect of increased beam currents, which increases the number of electrons per pixel without increasing the dwell time.

Biological images acquired on the Helios 650 dual beam system from FEI were used for evaluation. The average dwell time per pixel was kept constant at $10 \mu \mathrm{s}$ and each strategy was compared against a ground truth image with a dwell time of $40 \mu \mathrm{s}$ applying different figures of merit, such as Peak Signal to Noise Ratio (PSNR) and Structural Similarity Index (SSIM) [5]. Increasing beam current had no positive effect in any strategy. Denoising and SR both increased the quality of acquired data, however, the effect was not very convincing for both. A clearly different result was obtained with random sparse scanning followed by inpainting as reconstruction technique. BPFA was clearly superior to the other methods, which was supported by both the evaluation measures and the visual inspection of small structures (Figure 1). An important observation was, that the quality of the dictionary used in the inpainting algorithms is crucial for good results. BPFA learns a dictionary adapted to each reconstruction it performs, which makes the dictionary optimal in each case. However, this leads to a 
quite slow reconstruction. Contrary, EBI is based on prior data, so that the quality of training data determines the final reconstruction quality.

We conclude that the conventional approach of image acquisition should be challenged. Sparse acquisition techniques should have a much larger role in future microscopy, particularly for situations such as very large field-of-view scanning and three-dimensional acquisitions of samples, where the total acquisition time and electron dose are limiting factors [6].

\section{References:}

[1] S. Hawe et al, IEEE Trans. Image Process. 22(6) (2013), p. 2138.

[2] S. Villena et al, IEEE Intern Conf. on Image Process. (2010), p. 893.

[3] A. Stevens et al, Microscopy 63(1) (2014), p. 41.

[4] P. Trampert et al, Microsc. Microanal. 22(S3) (2016), p. 554.

[5] P. Trampert et al, "How should a fixed budget of dwell time be spent in scanning electron microscopy to optimize image quality?", Ultramicroscopy, under revision.

[6] Research has been supported by Thermo Fisher Scientific. The authors thank the DFKI GmbH for additional funding and for providing the necessary infrastructure.
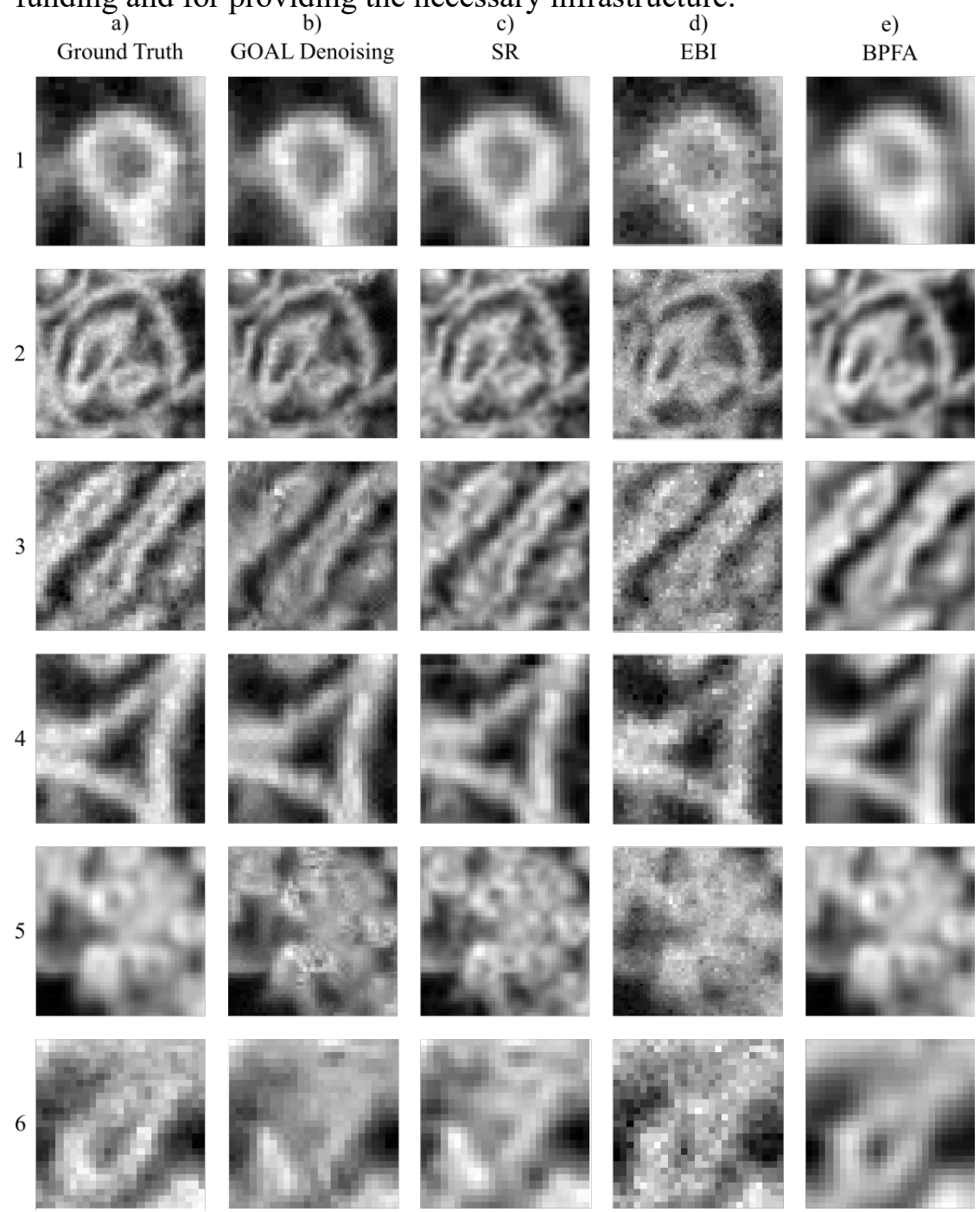

Figure 1. Impact of different strategies shown on some representative structures. a) Ground truth raster scan at $5 \mathrm{~nm}$ pixel size and $40 \mu \mathrm{s} d$ well time, b) strategy one: GOAL, c) strategy two: $S R, d$ ) strategy three: EBI, e) strategy three: BPFA. 